\title{
Damping Behavior of Incramute Modified by the Addition of Erbium TO ELIMINATE ROOM TEMPERATURE AgING
}

\author{
B.A. Ross and D.C. Van Aken \\ Department of Materials Science and Engineering \\ The University of Michigan \\ Ann Arbor, MI 48109-2136 \\ (Received August 21, 1989) \\ (Revised September 29, 1989)

\section{Introduction}

Incramute is a high damping $\mathrm{Mn}-\mathrm{Cu}$ alloy with a nominal composition (all compositions in weight percent) of $45 \mathrm{Mn}-53 \mathrm{Cu}-2 \mathrm{Al}(1)$. This alloy exhibits a specific damping capacity (SDC) as high as $68 \%(1,2)$ where the SDC is defined as the ratio of the energy lost to the maximum vibrational energy stored per cycle per unit volume. The high damping character of Incramute is formed by a solution heat treatment and aging sequence which develops a chemically modulated microstructure with Mn-rich regions. This microstructure exhibits the tweed morphology when imaged using transmission electron microscopy (TEM) and a two-beam diffraction contrast. The Mn-rich regions are believed to be unstable and exhibit a reversible martensitic transformation from face centered cubic (fcc) to body centered tetragonal (bct) (3). The bct transformation product is usually referred to as face centered tetragonal (fct) in the literature because the c/a ratio is near unity.

Much of the present understanding of this commercial alloy is based upon studies of the Mn-rich alloys, e.g. 70Mn$30 \mathrm{Cu}(4-7)$. Heat treatment of the Mn-rich materials is accomplished by forming a $\gamma$-fcc solid solution at high temperature and quenching to maintain the metastable $\gamma$ structure at room temperature (7-9). The stability of the $\gamma$-phase is dependent upon alloy composition, and $\mathrm{Cu}$ additions of greater than $20 \%$ are required to maintain this room temperature stability. The $\gamma$-phase is paramagnetic in the quenched state, but when artificially aged, the solid solution decomposes by a spinodal reaction (11) and a tweed microstructure results $(9,11-13)$. This decomposition has been studied by several investigators and a metastable $\gamma-(\mathrm{Mn}, \mathrm{Cu})$ miscibility gap has been established (11). Upon subsequent quenching the $\mathrm{Mn}$-rich $\gamma$-phase transforms martensitically to a highly twinned, antiferromagnetic fct structure $(8,9)$. In the fct unit cell, the magnetic spin of the $\mathrm{Mn}$ atoms is aligned parallel with the c-axis and an anti-parallel arrangement of spin produces the antiferromagnetic state (10). The twinned crystals are related by a simple twinning relationship on the fct (101) planes producing a near 90 degree rotation of the $c$ axis across the twin plane. Thus, the twin boundary separates two antiferromagnetic domains and it is believed that the high SDC results from the motion of these twin boundaries.

The Incramute alloys do not exhibit the highly twinned martensitic structure, but rather a chemically modulated structure exhibiting the tweed diffraction contrast. Perkins et al. have also observed a "flickering" diffraction contrast within the tweed structure $(2,3)$. This "flickering" contrast has been associated with small martensitic domains and the "flickering" resulting from a near $90^{\circ}$ rotation of the c-axis of the fct martensite. It should be noted that the size of these martensitic domains are of the order of $20 \mathrm{~nm}$. The addition of Sn to Incramute has been shown to promote a more general martensitic reaction (15).

All of the high damping $\mathrm{Cu}-\mathrm{Mn}$ alloys exhibit a loss of damping capacity when stored at room temperature. The values of SDC may drop to less than $10 \%$ after $100 \mathrm{hrs}$. storage at $19^{\circ} \mathrm{C}(7)$. This loss of SDC after room temperature storage, or aging, has many interpretations $(6,11)$ but all conclude that the microstructure becomes pinned. Butler and Kelly (6) explain the loss of SDC by the appearance of a subdomain structure in the martensite and Vitek and Warlimont (11) explain the loss of SDC by solute segregation to the twin-domain walls of the martensitic structure. Ritchie, Sprungman, and Sahoo have suggested that the room temperature aging is associated with diffusion of $\mathrm{C}$ or $\mathrm{Si}$, or both, to the antiferromagnetic domain walls (15). Thus, the room temperature aging may be a form of strain aging with the domain walls acting as solute traps. In the case of Incramute, the interstitial solute may become trapped at the strain centers associated with the tweed structure, thus stabilizing the Mn-rich regions. 
The purpose of this investigation was to determine if these pinning agents may be eliminated by precipitation of stable rare earth compounds. It is doubtful that $\mathrm{Si}$ has a sufficient mobility to produce the strain aging effect at room temperature and the present investigation will focous on the interstitial solutes $\mathrm{C}, \mathrm{O}$, and $\mathrm{N}$. The rare earth element Erbium (Er) was chosen for this work.

\section{Experimental Procedure}

Commercially produced Incramute with a nominal composition of $45 \mathrm{Mn}-53 \mathrm{Cu}-2 \mathrm{Al}$ (weight percent) was obtained from Olin Brass Co. via Caterpillar, Inc. The stock was received in the form of $6.4 \mathrm{~mm}$ thick sheet. Erbium additions of 0.1 weight percent were made to the wrought Incramute by induction melting under an Ar gas atmosphere using an alumina crucible. The $60 \mathrm{~g}$ molten charge was solidified under vacuum $\left(10^{-3}\right.$ torr $)$ and then water quenched to room temperature. The wrought Incramute and the Er-treated ingot were hot rolled at $800^{\circ} \mathrm{C}$ to produce a final sheet thickness between 1 to $1.5 \mathrm{~mm}$. Rectangular-beam test coupons having nominal dimensions of $50 \mathrm{~mm} \times 10 \mathrm{~mm} \times 1 \mathrm{~mm}$ were sheared from the central area of the rolled sheets. A solution heat treatment was performed at $820^{\circ} \mathrm{C}$ for 2 hours and was followed by a water quench. Artificial aging was performed at $400^{\circ} \mathrm{C}$ in two steps: the samples were first aged 4 hours to test an underaged condition; the samples were then aged an additional 12 hours to produce the peak-aged condition. The peak-aging time for Incramute was established by Reskusich as $16 \mathrm{~h}$ at $400^{\circ} \mathrm{C}(16)$. Both solution and aging heat treatments were performed under a positive pressure of argon gas and were subsequently water quenched. Aged samples were stored at $-25^{\circ} \mathrm{C}$ until testing to prevent room temperature aging.

A Dupont model 983 Dynamic Mechanical Analyzer (DMA) was used to measure the room temperature damping response as a function of strain amplitude, frequency, and time. The strain amplitude was varied from 200 to $2000 \mu \mathrm{\varepsilon}$ by changing the oscillation amplitude while a multiplexing program utilized test frequencies of $0.1,1.0,5.0$, and $10.0 \mathrm{~Hz}$. The strain stepping test (breakaway test) utilized a fixed frequency of $1 \mathrm{hz}$ and stepped the strain in a sequence of low-high-low, e.g. 450-1500-450 $\mu \varepsilon$.

Specimens for TEM were punched from the rolled sheet which had been thinned to $150-200 \mu \mathrm{m}$ using 600 grit SiC paper. Electrolytic thinning was performed using a Fischione Twin Jet polisher. Optimum thinning and polishing were obtained with an electrolyte containing (by volume) $67 \%$ ethanol, and $33 \%$ nitric acid using a potential of $20 \mathrm{~V}$ and a current density of approximately $6 \mathrm{~mA} / \mathrm{mm}^{2}$. Surface film formation resulted after removal of the sample from the electrolyte and this was subsquently removed by ion milling [Ar] for 20 minutes.

\section{$\underline{\text { Results }}$}

Solution heat treatment of the wrought and the Er-treated material produced an average grain size of $100 \mu \mathrm{m}$, see Fig. 1. Annealing twins, typical of $\mathrm{Cu}$-based fcc materials, were evident in each material. Initial damping tests were performed on the underaged samples by examining the strain dependence of the loss tangent and room temperature aging. Tests were conducted at a frequency of $1 \mathrm{~Hz}$ and between 200 and $2000 \mu \varepsilon$. The strain amplitude dependence of the damping is shown in Fig. 2 and the effect of room temperature aging is summarized in Table I. Both materials exhibited room temperature aging in the underaged condition, but it should be noted that the loss tangent of the Ermodified Incramute shows a higher strain amplitude dependence. In addition, the underaged samples were subjected to a strain stepping experiment to determine if the application of a high strain amplitude would restore the room temperature aged material to a higher damping state, see Fig. 3. Indeed, some of the damping capacity was recovered in the wrought and the Er-modified materials. It should also be noted that the loss tangent for the wrought material decreases with strain amplitudes greater than $1000 \mu \varepsilon$. This decrease in the damping with the higher strain amplitudes has been observed by other workers $(5,7,12,16)$ in the underaged $\mathrm{Cu}-\mathrm{Mn}$ materials.

The underaged samples were then aged an additional 12 hours at $400^{\circ} \mathrm{C}$ to produce the peak-aged condition. The strain amplitude dependence of the loss tangent is shown in Fig. $2 \mathrm{~b}$ for both materials. Again the Er-modified Incramute shows a higher strain amplitude dependence for the loss tangent. The loss of damping as a result of room temperture aging is summarized in Table I. As may be observed from these results the Er-modified material does not show a significant room temperature aging dependence. After 264 hours of storage at $23^{\circ} \mathrm{C}$ the peak-aged $0.1 \% \mathrm{Er}$ sample displayed a loss tangent of 0.029 when measured at a frequency of $1 \mathrm{~Hz}$ and a strain amplitude of $490 \mu \varepsilon$. The loss in damping capacity resulting from room temperature aging was only $5 \%$. This may be compared to a $45 \%$ decrease observed for the wrought Incramute sample. Furthermore, the strain stepping experiments show that the wrought Incramute loses damping very rapidly even at the high strain amplitudes, while the Er-modified Incramute is relatively insensitive, see Fig. 4. A comparison of the peak-aged alloys (Fig. 5) shows a qualitative difference in the tweed microstructure between the wrought and Er-modified materials. Both TEM bright-field images were recorded with $\mathbf{g}=022$ and the electron beam aligned close to $<011>$. Although the magnitude of the tweed modulation is similiar 
between the two materials, the orientation of the tweed contrast differs significantly. In addition, a Mn-rich second phase is present in the Er-modified alloy and this second phase contains Si as shown by $\mathrm{x}$-ray energy dispersive spectroscopy (XEDS), see Fig. 6. A complete TEM analysis of the two materials will be presented elsewhere (18).

\section{Discussion}

The most significant observations from this investigation were that the addition of $0.1 \%$ erbium to Incramute substantially reduced the loss of damping capacity associated with room temperature aging and increased the strain dependence of the loss tangent. Therefore, it may be concluded that the damping capacity of Incramute can be considerably improved by micro-alloying with erbium. It is interesting to note that previous work concerning rare earth additions ( $\mathrm{Sm}$ and $\mathrm{Yb}$ ) to Incramute did not produce significant changes in the damping properties (19). In these earlier studies, however, Er was not used and the rare earth additions were made to a Sn-modified Incramute designated as Incramute II.

These initial studies of the wrought and Er-modified Incramute suggest a strain aging effect is responsible for the loss in damping capacity during room temperature storage. This type of mechanism has been previously suggested by Richie et al. for the room temperature aging of the Sonoston alloy (15). Sonoston has a nominal composition given in weight percent as $55 \mathrm{Mn}-37 \mathrm{Cu}-4 \mathrm{Al}-3 \mathrm{Fe}-1 \mathrm{Ni}$. In the Sonoston alloy, the investigators speculated that interstials (C,O, and $\mathrm{N}$ ) or $\mathrm{Si}$, or both were responsible for the damping capacity loss. Examination of the room temperature diffusivity of $\mathrm{Si}$ in $\mathrm{Cu}$ would suggest that $\mathrm{Si}$ is not responsible for the loss of damping which is observed on short time scales, i.e. less than 24 hrs., but may account for damping losses which result after 7 days. Indeed the strain aging associated with Si may be more detrimental since Si would be a more effective pinning agent. Hence, Si diffusion and subsequent solute pinning may explain the long-term loss of damping capacity while short term losses may still be attributed to solute pinning by interstitials.

A qualitative understanding of the observed strain stepping behavior may be illustrated by considering the difference in the number of interstitial solute atoms per unit area of active damping interface for the underaged and peakaged conditions. Assuming that both the wrought and Er-modified Incramute samples respond to aging in a similar fashion, i.e. the same aging kinetics, then equivalent damping microstructures are developed. Thus, an equal density of damping interface would exist in the two materials studied. Artificial aging, up to the peak-aged condition ( 16 hrs. $x$ $400^{\circ} \mathrm{C}$ ), would increase the twin-domain interface area. The erbium-rich dispersoids are thought to tie-up Si and some interstitial atoms, thereby reducing the number of available pinning agents. The observed breakaway behavior (see Figs. $4 \mathrm{a} \& 4 \mathrm{~b}$ ) in the wrought and $0.1 \%$ Er Incramute samples may then be explained by using the interstitial density argument given above. Thus, the Er modified Incramute is not subject to strain aging in the peak-aged condition, but exhibits some strain aging when the available twin-domain interface area is smaller as expected in the underaged condition.

It is interesting to speculate that the erbium serves a dual purpose in improving the damping character of Incramute. Firstly, the presence of the Mn-Er second phase demonstrates a gettering of $\mathrm{Si}$ and possibly interstitials. Analytical electron microscopy studies of the Er-modified Incramute will be presented elsewhere (18). As stated previously by Ritchie et. al., Si may have a detrimental effect on the room temperature damping stability (15). Thus, the removal of Si from solid solution would eliminate one possible pinning agent. In addition, Si may be a more effective pinning agent than the interstitials, thus being more detrimental to the overall damping performance of Incramute. The second effect of the Er addition may be related to the electron/atom variation of the Mn-rich regions in the aged structure. The presence of $\mathrm{Er}$, or perhaps the absence of $\mathrm{Si}$, may inerease the electron/atom ratio thus contributing a larger change in the magnetic moment when the c-axis is reorientated, as observed by Perkins et al. (2-3). Thus, the loss tangent would show a higher strain dependence.

\section{Conclusions}

The addition of 0.1 weight percent Er to Incramute has been shown to increase the strain dependent damping and to significantly reduce the room temperature aging behavior. The room temperature aging phenomenon appears to be a strain aging effect. Erbium forms a Mn-rich second phase and acts as a getter for Si and possibly interstitials. The loss of damping capacity during room temperature aging may occur in two stages. A short term component may occur on the time scale of minutes involving the diffusion and interface pinning by interstitial solute atoms and strain stepping experiments demonstrated a break-away behavior (increase in loss tangent) analogous to the Lechatlier effect. The damping capacity loss occurring on a time scale of weeks may be the result of interface pinning by $\mathrm{Si}$ which would be expected to be more detrimental producing a permanent loss of damping. 


\section{Acknowledgements}

This work has been funded in part by the Office of Naval Research and the David Taylor Research Center under contract 87-K-0452. The technical monitor was Dr. D.E. Polk. The electron microscopy studies were performed at the Electron Microbeam Analysis Laboratory at the University of Michigan.

\section{References}

1. Incramute Technical Data Sheet, International Copper Research Association, Inc. (1976).

2. J. Perkins, L.L. Mayes, and T. Yamashita, Scripta Metall., 22, 887 (1988).

3. J. Perkins, L.L. Mayes, and T. Yamashita, Scripta Metall., 22, 1137 (1988).

4. D. Birchon, Eng. Mater. Design, 1, 692 (1964).

5. D. Birchon, D.E. Bromley, and D. Healy, Metal Science J., 2,41 (1968).

6. E.P. Butler and P.M. Kelly, Trans. TMS-AIME, 242, 2107 (1968).

7. R.J. Goodwin, Metal Sci. J., 2, 121 (1968).

8. Z.S. Basinski and J.W. Christian, J. Inst. Metals, 80,659 (1952).

9. E.P. Butler and P.M. Kelly, Trans. TMS-AIME, 242, 2099 (1968).

10. D. Meneghetti and S.S. Sidhu, Phys. Rev., 105, 2099 (1968).

11. J.M. Vitek and H. Warlimont, Metal Science, January, 7 (1976).

12. J.A. Hedley, Metal Sci. J., 2, 129 (1968).

13. I.E. Anderson and B.B. Rath, in Rapidly Solidified Crystalline Alloys, (edited by S.K. Das, B.H. Kear, and C.M. Adam) The Metallurgical Society, Inc. (1985), p.219.

14. McD. Schetky and D.T. Peters, Role of Interfaces on Material Damping, (eds. B.B. Rath and M.S. Misra) ASM (1985), p.67.

15. I.G. Ritchie, K.W. Sprungmann, and M. Sahoo, J. de Phys., 46, C10- 409 (1985).

16. J. Reskuvich, Masters thesis, Naval Postgraduate School, Monterey California, Sept. 1986.

17. International Copper Research Association, Report \# 274 (1972).

18. B.A. Ross and D.C. Van Aken, to be published.

19. International Copper Research Association, Report \# 221 (1976).

\section{TABLE I}

Loss tangent values measured as a function of room temperature aging time at $1 \mathrm{hz}$ and $\sim 470 \mu \varepsilon$.

\begin{tabular}{|c|c|c|c|c|}
\hline \multirow{2}{*}{ Time $(\mathrm{t})$} & \multicolumn{2}{|c|}{ Underaged $\left(4 \mathrm{~h} \times 400^{\circ} \mathrm{C}\right)$} & \multicolumn{2}{c|}{ Peak-aged $\left(16 \mathrm{~h} \times 400^{\circ} \mathrm{C}\right)$} \\
\cline { 2 - 5 } & Wrought & $\begin{array}{c}0.1 \% \mathrm{Er} \\
\text { Modified }\end{array}$ & Wrought & $\begin{array}{c}0.1 \% \mathrm{Er} \\
\text { Modified }\end{array}$ \\
\hline $10 \mathrm{~min}$. & 0.0156 & 0.0191 & 0.0197 & 0.0304 \\
\hline $30 \mathrm{~min}$. & 0.0151 & 0.0185 & 0.0190 & 0.0300 \\
\hline $60 \mathrm{~min}$. & 0.0146 & 0.0181 & 0.0180 & 0.0298 \\
\hline $2 \mathrm{hrs}$. & 0.0136 & 0.0175 & & \\
\hline $4 \mathrm{hrs}$. & 0.0128 & 0.0165 & & 0.0287 \\
\hline 8 hrs. & 0.0119 & 0.0152 & & 0.0277 \\
\hline 7 days & 0.009 & 0.0140 & & \\
\hline 11 days & & & 0.0107 & 0.0290 \\
\hline
\end{tabular}



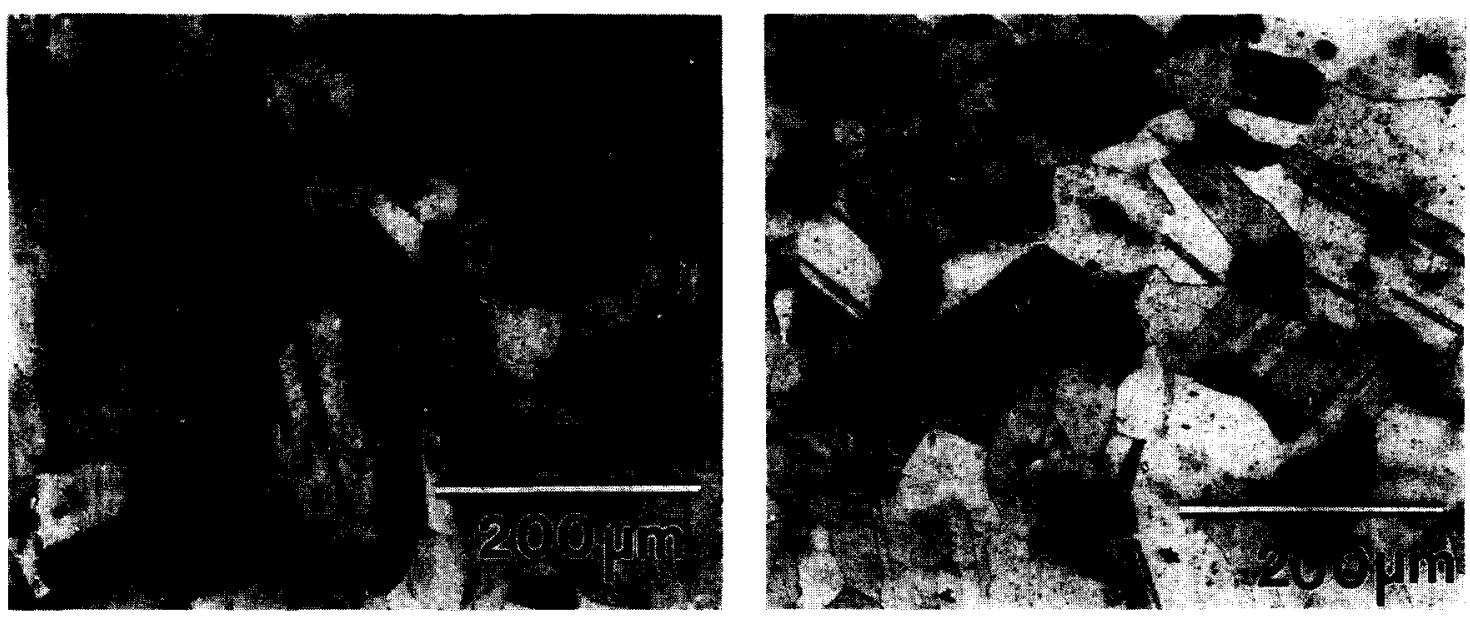

Fig. 1. Optical micrographs of (a) wrought Incramute and (b) $0.1 \%$ Er-modified Incramute. Alloys were solution treated at $820^{\circ} \mathrm{C}$ for $2 \mathrm{hrs}$. and subsequently water quenched.
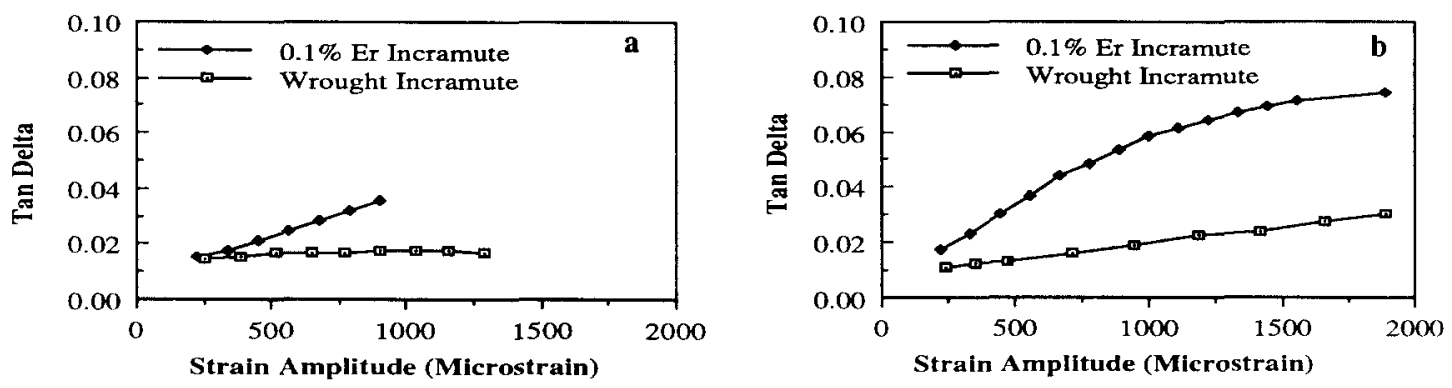

Fig. 2. Plots of the loss tangent (tan delta) measured as a function of strain amplitude for wrought Incramute and Ermodified Incramute alloy. (a) Aged $4 \mathrm{hrs} . \times 400^{\circ} \mathrm{C}$ and (b) Aged $16 \mathrm{hrs}$. $400^{\circ} \mathrm{C}$.
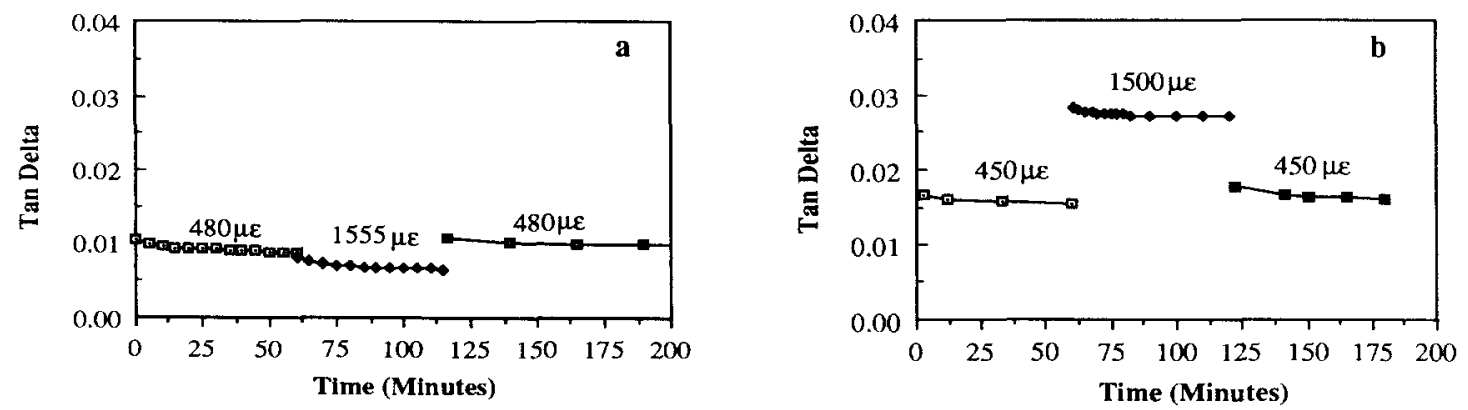

Fig. 3. Loss tangent vs. time plots measured on underaged samples during a strain stepping program. (a) wrought Incramute and (b) $0.1 \%$ Er modified Incramute. 

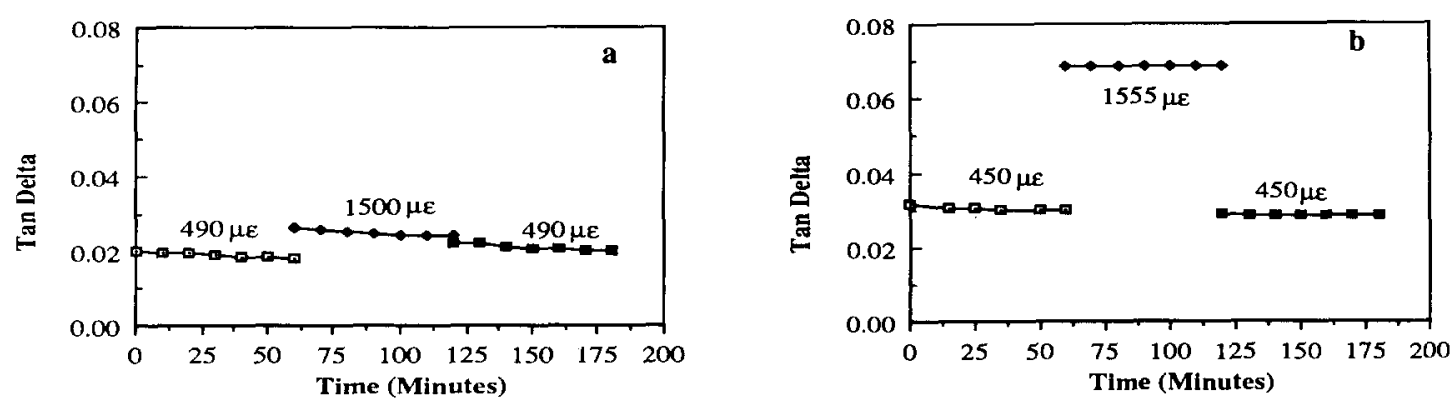

Fig. 4. Loss tangent vs. time plots of the peak aged alloys. (a) wrought Incramute and (b) $0.1 \%$ Er alloy.
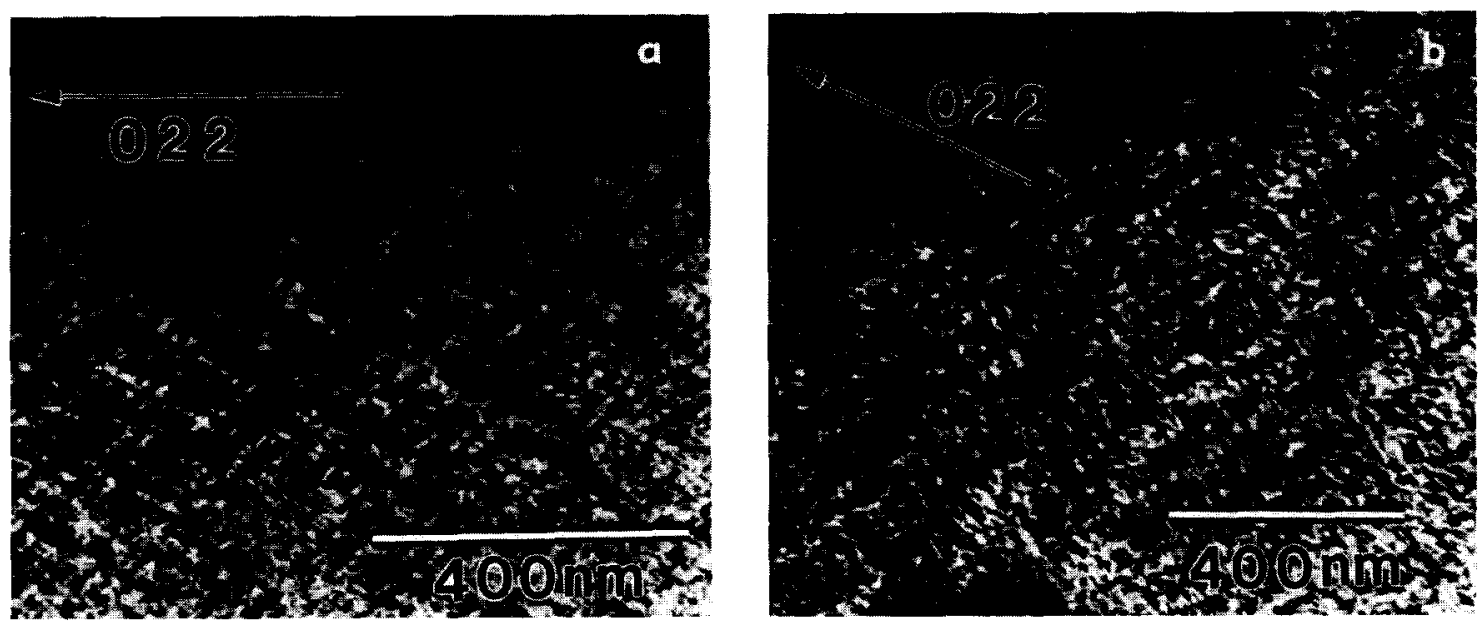

Fig. 5. Bright-field transmission electron micrographs of peak-aged (a) wrought Incramute and (b) $0.1 \%$ Er-modified Incramute. The electron beam direction for both images was near $\mathrm{a}<011>$ and $\mathbf{g}=022$.

Fig. 6. XEDS of peak aged $0.1 \%$ Er modified Incramute alloy. The probe was focused on a second phase particle.

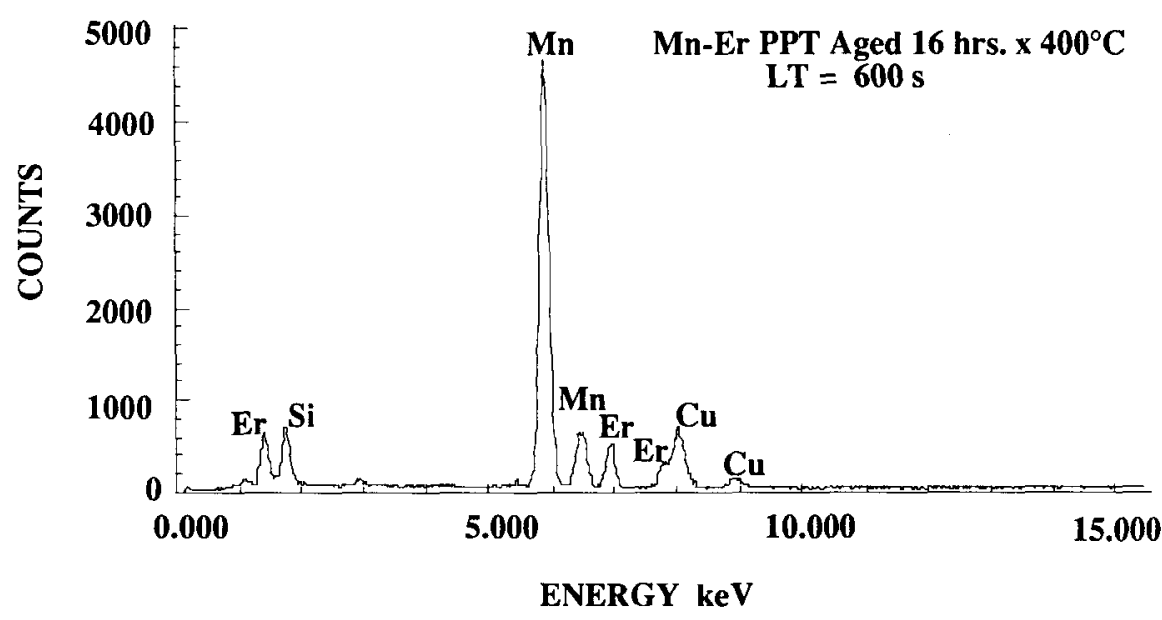

